\title{
NOT EVERY MINIMAL HAUSDORFF SPACE IS $e$-COMPACT
}

\author{
R. M. STEPHENSON, JR.
}

ABSTRACT. A topological space $X$ is said to be $e$-compact with respect to a dense subset $D$ provided either of the following equivalent conditions is satisfied: (i) every open cover of $X$ has a finite subcollection which covers $D$; (ii) every ultrafilter on $D$ converges to a point of $X$. If there exists a dense subset with respect to which a space $X$ is $e$-compact, then $X$ is called $e$-compact. ${ }^{1}$

Two problems recently raised by S. H. Hechler are the following. (a) Is every minimal Hausdorff space e-compact? (b) If there exists a Hausdorff space which is $e$-compact with respect to a space $D$, must $D$ be completely regular? The main purpose of this paper is to provide a negative answer to (a) and to present some results which the author hopes will be of use in the solution to (b). These results can also be used to obtain a construction of $\beta X$ for certain completely regular Hausdorff spaces $X$.

1. Introduction. A Hausdorff space $X$ is said to be absolutely closed (e.g., see [BPS, p. 97]) if any of the following equivalent conditions holds: (i) for every open cover $\mathcal{U}$ of $X$, there is a finite subcollection $\mathcal{F}$ of $\mathcal{U}$ such that $X=(\cup \mathcal{F})^{-}$; (ii) for every open filter base $\mathcal{G}$ on $X$, the adherence of $\mathcal{G}$, $\bigcap\{\bar{G} \mid G \in \mathcal{G}\}$, is nonempty; (iii) $X$ is a closed subspace of every Hausdorff space in which it can be embedded.

A Hausdorff space on which there exists no strictly weaker Hausdorff topology is called minimal Hausdorff. In [K] Katětov proved that a Hausdorff space is minimal Hausdorff if and only if it is absolutely closed and semiregular. (A space $X$ is called semiregular provided that the topology which has the family of regular open subsets of $X$ as a base is the same as the given topology; a subset $V$ of $X$ is regular open if $V=\operatorname{Int}(\mathrm{Cl} V)$.)

In $[H]$ Hechler proved that a Hausdorff space. $X$ is e-compact with respect to a dense subset $D$ if and only if $X$ is absolutely closed and has the following property: for each point $p \in X$ and open neighborhood $V$ of $p$ there exists an open neighborhood $W$ of $p$ such that $\bar{W} \cap D \subset V \cap D$. Using the denseness of $D$, one can restate this result as follows.

Theorem 1.1 (S. H. Hechler). Let $X$ be a Hausdorff space and $D a$ dense subset of $X$. The following are equivalent.

Received by the editors July 3, 1974. $54 \mathrm{G} 20$.

AMS(MOS) subject classifications (1970). Primary 54D25, 54D30; Secondary

Key words and phrases. Stone-Čech compactifications, Banaschewski minimal Hausdorff completions, absolutely closed spaces, $e$-compact spaces.

${ }^{1}$ This concept is not the same as S. Mrowka's notion of E-compact. 
(i) $X$ is e-compact with respect to $D$.

(ii) $X$ is absolutely closed, and for every point $p$ of $X$, the space $D \cup\{p\}$ is regular.

The next theorem gives a relationship between minimal Hausdorff spaces and $e$-compact spaces.

We recall that a space is said to be Urysohn if every pair of distinct points have disjoint closed neighborhoods.

Theorem 1.2. Let $X$ be a semiregular Hausdorff space and $D$ a dense subset of $X$. The following are equivalent.

(i) $X$ is e-compact with respect to $D$.

(ii) $X$ is absolutely closed, and for every pair of points $p, q$ of $X$, the space $D \cup\{p, q\}$ is Urysohn.

Proof. (i) implies (ii). If (i) holds, then, clearly, for any compact subset $K$ of $X$, the space $X$ is $e$-compact with respect to $D \cup K$. Thus by Theorem 1.1, $X$ is absolutely closed, and the space $D \cup\{p, q\}$ is regular and hence Urysohn.

(ii) implies (i). Let $\mathcal{U}$ be an ultrafilter on $D$. We will prove that $\mathcal{U}$ converges to a point of $X$ 。

Let $\mathcal{P}=\{P \subset X \mid P$ is open and $P \cap D \in \mathcal{U}\}$. Since $\mathcal{P} \mid D \subset \mathcal{U}$, it suffices for us to prove that $\mathcal{P}$ converges to a point of $X$. Now on a minimal Hausdorff space it is known [Bo] that any open filter base with at most one adherent point is convergent. Let us then show that $\mathcal{P}$ has at most one adherent point.

Choose any two points $p, q \in X$, and select open neighborhoods $V$ and $W$ of $p$ and $q$ so that for $E=D \cup\{p, q\}$, the sets $K=\mathrm{Cl}_{E}(V \cap E)$ and $L=$ $\mathrm{Cl}_{E}(W \cap E)$ are disjoint. Because $\mathcal{U}$ is an ultrafilter, exactly one of the sets $K \cap D, L \cap D$, and $D \backslash(K \cup L)$ must belong to $\mathcal{U}$. If $D \backslash(K \cup L) \in \mathcal{U}$ and $T$ is any open subset of $X$ such that $T \cap D=D \backslash(K \cup L)$, then $T \in \mathcal{P}$ and it follows from the denseness of $D$ that $T \cap V=\varnothing=T \cap W$, so that neither $p$ nor $q$ is in $\bigcap\{\bar{P} \mid P \in \mathcal{P}\}$. Similarly, if $K \cap D \in \mathcal{U}$ and $S$ is an open subset of $X$ such that $S \cap D=D \backslash L$, then $S \in \mathcal{P}$ and $S \cap W=\varnothing$, so that $q$ is not an adherent point of $\mathcal{P}$.

Remark 1.3. The following space shows that condition (ii) of Theorem 1.2 is not equivalent with condition (ii) of Theorem 1.1.

Let $I=[0,1]$ and let $\mathcal{T}$ be the weakest topology on $I$ which contains $\mathbf{Q} \cap I$ and the usual open subsets of $I$. Then for $D=I$, condition (ii) of Theorem 1.2 is satisfied, but (it is well known and easy to prove that) the space $(I, \mathcal{T})$ is absolutely closed and not compact, so $(I, \mathfrak{T})$ is not e-compact with respect to $D$.

2. A non-e-compact minimal Hausdorff space. In order to present the main example, we will use the following result (obtained in [Ba]) and notation. 
Construction 2.1. Let $X$ be a semiregular Hausdorff space. A filter base $\mathfrak{F}$ on $X$ is said to be a maximal semiregular filter on $X$ if $\mathcal{F}$ is a maximal family of nonempty regular open subsets of $X$ such that whenever $F \in \mathcal{F}$ and $G \in \mathcal{F}$ then $F \cap G \in \mathcal{F}$. Let $\mathbf{T}(X)=\{\mathfrak{F} \mid \mathcal{F}$ is a maximal semiregular filter on $X$ having empty adherence $\}$, and for a regular open subset $V$ of $X$, let $V^{*}=V \cup\{\mathfrak{F} \in \mathbf{T}(X) \mid V \in \mathcal{F}\}$. We will denote by $B(X)$ the set $X \cup \mathbf{T}(X)$, with the topology which has as a base $\left\{V^{*} \mid V\right.$ is a regular open subset of $\left.X\right\}$. Then $X$ is a dense subspace of $B(X)$ and $B(X)$ is a minimal Hausdorff space [Ba].

Theorem 2.2. Let $X$ be a locally compact Hausdorff space such that for each point $p$ of $X$ there is a regular open subset of $X$ having $p$ as a boundary point. Suppose also that there exist an infinite cardinal $A$ and a one-toone listing $\left\{W_{a} \mid a \in A\right\}$ of the members of a pairwise disjoint family of nonempty open subsets of $X$ such that for each point $p \in X$ there exists $b \in A$ with $p \notin \in\left(\bigcup\left\{w_{a} \mid b \leq a\right\}\right)^{-}$.

Then the Banaschewski minimal Hausdorff completion $B(X)$ of $X$ fails to be e-compact.

Proof. Since $X$ is a dense locally compact subset of the Hausdorff space $B(X), X$ must also be open in $B(X)$. Thus for any dense subset $E$ of $B(X), E \cap X$ is dense in $B(X)$, and obviously if $B(X)$ is e-compact with respect to $E$, then it is also e-compact with respect to $E \cap X$. Let us then consider an arbitrary dense subset $D$ of $X$ and prove that $B(X)$ is not $e$ compact with respect to $D$.

For each $a \in A$ choose a point $d_{a} \in D \cap W_{a}$ and a regular open subset $V_{a}$ of $X$ with $d_{a} \in\left(\mathrm{Cl}_{X} V_{a}\right) \backslash V_{a}$ and with $V_{a} \subset \mathrm{Int}_{X}\left(\mathrm{Cl}_{X} W_{a}\right)$ (if $R$ is a regular open subset of $X$ having $d_{a}$ as a boundary point, take $V_{a}=R \cap$ Int $\left.{ }_{X}\left(\mathrm{Cl}_{X} W_{a}\right)\right)$. Let $I=\left\{d_{a} \mid a \in A\right\}$ and $C$ be the family of all open subsets $V$ of $X$ such that $I \subset V \subset \bigcup\left\{W_{a} \mid a \in A\right\}$. For each $b \in A$ and $V \in \mathcal{C}$, define

$$
s(b, V)=\operatorname{Int}_{X}\left(\mathrm{Cl}_{X}\left(V \cap\left(\bigcup\left\{V_{a} \mid b \leq a\right\}\right)\right)\right) .
$$

Let $\mathcal{S}=\{S(b, V) \mid b \in A$ and $V \in \mathcal{C}\}$.

By Zorn's lemma there exists a maximal semiregular filter $\mathcal{U}$ on $X$ such that $\mathcal{S} \subset \mathcal{U}$. By hypothesis for any point $p \in X$ there exists $b \in A$ for which $p \notin \mathrm{Cl}_{X}\left(\bigcup\left\{W_{a} \mid b \leq a\right\}\right)$, and from this it follows that $p \notin$ $\mathrm{Cl}_{X} S\left(b, \bigcup\left\{w_{a} \mid a \in A\right\}\right)$. Thus

$$
\bigcap\left\{C 1_{X} U \mid U \in \mathcal{U}\right\} \subset \bigcap\left\{\mathrm{Cl}_{X} S \mid S \in \delta\right\}=\varnothing,
$$

so $U \in \mathbf{T}(X)$.

We will complete the proof that $B(X)$ is not e-compact with respect to $D$ by showing that in the space $E=D \cup\{\mathcal{U}\}$, the point $\mathcal{U}$ is not in the closed set $C=\mathrm{Cl}_{E} I$, but $U$ and $C$ are not contained in disjoint open sets. 
In order to show that $\mathcal{U} \notin C$, it suffices to prove that the set $S=$ Int ${ }_{X}\left(\mathrm{Cl}_{X}\left(\bigcup\left\{V_{a} \mid a \in A\right\}\right)\right)$ satisfies $S \cap I=\varnothing$, for from the relations $I \subset D \subset$ $X$ and $S \in \mathcal{S} \subset \mathcal{U}$, it follows that the basic open set $S^{*}$ satisfies $\mathcal{U} \in S^{*}$ and $S^{*} \cap I=S \cap I$.

Consider an arbitrary point $d_{b}$. If $d_{b} \in S$ then there must exist an open subset $T$ of $X$ with $d_{b} \in T \subset \mathrm{Cl}_{X}\left(\bigcup\left\{V_{a} \mid a \in A\right\}\right)$. Since

$$
\mathrm{Cl}_{X}\left(\bigcup\left\{V_{a} \mid a \in A\right\}\right)=\mathrm{Cl}_{X} V_{b} \cup \mathrm{Cl}_{X}\left(\bigcup\left\{V_{a} \mid a \neq b\right\}\right)
$$

and $W_{b}$ is an open subset of $X$ such that $W_{b} \cap\left(\bigcup\left\{V_{a} \mid a \neq b\right\}\right)=\varnothing$, it follows that $W_{b} \cap T$ is an open neighborhood of $d_{b}$ in $X$ which is contained in $\mathrm{Cl}_{X} V_{b}$. Thus $d_{b} \in \mathrm{Int}_{X}\left(\mathrm{Cl}_{X} V_{b}\right)=V_{b}$, in contradiction of the fact that $d_{b}$ is a boundary point of $V_{b}$. Therefore, $d_{b}$ cannot be in $S$, and $S \cap I=\varnothing$.

Suppose next that $J, K$ are arbitrary open subsets of $E$ such that $I \subset J$ and $\mathcal{U} \in K$. We wish to prove that $J \cap K \neq \varnothing$.

There exist sets $V \in \mathcal{C}$ and $U \subset X$ such that $V \cap E \subset J$ and $\mathcal{U} \in U^{*} \cap$ $E \subset K$. Then Int ${ }_{X}\left(\mathrm{Cl}_{X} V\right) \in \mathcal{U}$ and $U \in \mathcal{U}$, so $U \cap \operatorname{Int}_{X}\left(\mathrm{Cl}_{X} V\right) \neq \varnothing$. Since $U$ is an open subset of $X$, it must also be the case that $U \cap V \neq \varnothing$. Thus $\varnothing \neq U^{*} \cap V \cap E \subset J \cap K$, and that completes the proof.

If $X$ is a topological space in which each point has a neighborhood base that is totally ordered by the inclusion relation, then each nonisolated point $p$ of $X$ is a boundary point of some regular open subset $R$ of $X$. (For one can find a neighborhood base $\pi$ for $p$ well ordered by reverse inclusion and a smallest cardinal $C$ for which there exists a cofinal subset $M$ of $(\pi, \supset)$ with $C=$ card $M$; then if $\left\{M_{c} \mid c \in C\right\}$ is a one-to-one listing of the members of $\mathbb{M}$, one can use the minimality of $C$ to find a family $\left\{W_{c} \mid c \in C\right\} \subset M$ such that for all $c, d \in C,(1) c \leq d$ if and only if $W_{c} \supset W_{d}$, and (2) if $c \leq d$ then $W_{d} \subset M_{c}$. Next, since every cofinal subset of $C$ has cardinality $C$, one can find a family of pairwise disjoint nonempty open sets $\left\{V_{c} \mid c \in C\right\}$ such that each $V_{c} \subset W_{c}$; then for any $B \subset C$ such that $B$ and $C \backslash B$ are cofinal in $C$, it suffices to take $R=\operatorname{Int}_{X}\left(C l_{X}\left(\bigcup\left\{V_{c} \mid c \in B\right\}\right)\right)$.) Thus it follows from Theorem 2.2 that $B(X)$ fails to be e-compact for a space $X$ such as the long line or, say, any noncompact, locally compact, metrizable space without isolated points.

In case the set $I$ of isolated points of a space $X$ is dense in $X$, then obviously by Theorem 1.1 any absolutely closed extension space of $X$ is $e$-compact (with respect to $I$ ). Likewise, if no point of $X$ has a compact neighborhood, then one can use Theorem 1.2 and the equation $\mathrm{Cl}_{B(X)} V^{*}=$ $\mathrm{Cl}_{X} V \cup V^{*}$ to prove that $B(X)$ is e-compact with respect to the dense subset $B(X) \backslash X$.

3. e-compactifiable spaces. A topological space $D$ is called e-compactifiable if there exists a Hausdorff space $X$ which is e-compact with re- 
spect to $D$, and any Hausdorff space which is $e$-compact with respect to $D$ is said to be an e-compactification of $D$. In $[H]$ Hechler asked if every $e$ compactifiable space is completely regular, and he proved that the topological property e-compactifiable is productive and hereditary. A proof was also given that if a space $X$ is $e$-compact with respect to a set $D$ and if $f$ is a continuous mapping of $X$ into a Hausdorff space, then the space $f(X)$ is an e-compactification of $f(D)$.

While the author does not know the answer to the above question of Hechler, it is shown in this section that every $e$-compactifiable space $X$ has a maximum e-compactification $M(X)$ which behaves much like the StoneČech compactification of a completely regular Hausdorff space. Furthermore, a close relationship between $M(X)$ and $\beta X$ is established for certain completely regular Hausdorff spaces $X$.

The following terminology (due to $B$. Banaschewski) will be used. If a space $X$ is a dense subspace of a space $K$ (in which case $K$ is called an extension space of $X)$, then for each point $p \in K \backslash X, \mathcal{C}(p)=\{V \cap X \mid V$ is an open neighborhood of $p$ in $K\}$ is called the trace filter of $p$ on $X$. The family $\mathbf{T}$ of all trace filters on $X$ of points of $K \backslash X$ is said to be the filter trace of $K$ on $X$, and $K$ is called the simple extension of $X$ with filter trace $\mathbf{T}$ if: for every subset $V$ of $K, V$ is open if and only if (a) $V \cap X$ is open in $X$, and (b) $p \in V \backslash X$ implies $V \cap X \in \mathcal{C}(p)$.

An ultrafilter $\mathcal{U}$ on a subspace $X$ of some space will be called a nonconvergent ultrafilter on $X$ provided that $U$ has no adherent point in $X$.

Construction 3.1. Let $X$ be an e-compactifiable space and let $K(X)$ be the family of all e-compactifications of $X$. For each $K \in \mathcal{K}(X)$, let $\mathbf{T}(K)$ be the filter trace of $K$ on $X$. Next, for each nonconvergent ultrafilter $\mathcal{U}$ on $X$, define $\mathbf{E}(\mathcal{U})=\{\mathfrak{F} \in \mathrm{T}(K) \mid K \in \mathcal{K}(X)$, and $\mathcal{F} \subset \mathcal{U}\}$, and denote by $p(\mathcal{U})$ the family of all open subsets $V$ of $X$ such that for some finite subset $A$ of $\mathrm{UE}(\mathcal{U}), V \supset \bigcap A$. Let $\mathbf{T}=\{p(\mathcal{U} \mid \mathcal{U}$ is a nonconvergent ultrafilter on $X\}$. We will denote by $M(X)$ the set $X \cup \mathbf{T}$, topologized so that $M(X)$ is the simple extension of $X$ with filter trace $\mathbf{T}$.

Theorem 3.2. Let $X$ be an e-compactifiable space. Then the following hold.

(i) $M(X)$ is an e-compactification of $X$.

(ii) For every e-compactification $K$ of $X$, there exists a continuous mapping $f: M(X) \rightarrow K$ such that $f \mid X=i$, the identity on $X$.

(iii) Each point $p \in M(X) \backslash X$ is a prime open filter, i.e., if $A$ and $B$ are disjoint open subsets of $X$ with $A \cup B \in p$, then either $A \in p$ or $B \in p$.

Proof. (i) To see that $M(X)$ is Hausdorff, consider two points $x, y \epsilon$ $M(X)$. If both $x$ and $y$ are in the open set $X$, then they have disjoint neigh- 
borhoods in $X$ and hence also in $M(X)$. If $x \in X$ and $y=p(\mathcal{U})$, choose any $L \in \mathcal{K}(X)$ and point $t \in L$ to which $\mathcal{U}$ converges, and note that because $L$ is Hausdorff, there are disjoint open subsets $W$ and $V$ of $L$ such that $x \epsilon$ $W$ and $t \in V$. Then $V \cap X \in p(\mathcal{U})$, so $W \cap X$ and $(V \cap X) \cup\{p(\mathcal{U})\}$ are disjoint neighborhoods of $x$ and $y$ in $M(X)$. In case $x=p(\mathcal{U})$ and $y=p(\mathcal{Y})$, then since $x \neq y$, there must exist $K \in \mathcal{K}(X)$ and $\mathcal{F}, \mathcal{G} \in \mathbf{T}(K)$ such that $\mathcal{F} \subset \mathcal{U}$, $\mathcal{S} \subset \mathcal{C}$, and $\mathcal{F} \neq \mathcal{G}$. Appealing to the fact that $K$ is Hausdorff, one can choose disjoint sets $F \in \mathcal{F}$ and $G \in \mathcal{G}$. Then $F \in p(\mathcal{U})$ and $G \in p(\mathcal{C})$, and $F \cup\{p(\mathcal{U})\}$ and $G \cup\{p(\mathbb{O})\}$ are disjoint neighborhoods of $x$ and $y$.

By construction each nonconvergent ultrafilter $\mathcal{U}$ on $X$ converges to the point $p(\mathcal{U})$, so $M(X)$ is $e$-compact with respect to $X$.

(ii) Let $K \in \mathcal{K}(X)$. For each $x \in X$ set $f(x)=x$, and for a nonconvergent ultrafilter $\mathcal{U}$ on $X$, choose a point $y \in K \backslash X$ to which $\mathcal{U}$ converges and define $f(p(\mathcal{U}))=y$. Then $f \mid X=i$, and it is easy to see that $f: M(X) \rightarrow K$ is continuous.

(iii) Let $A_{1}, A_{2}$ be disjoint nonempty open subsets of $X$ with $A_{1} \cup$ $A_{2} \in p(\mathcal{U})$, and suppose that for $i=1,2, A_{i} \cap U \neq \varnothing$ for every $U \in p(\mathcal{U})$. Then $q\left(A_{i}\right)=\left\{V \mid V\right.$ is an open subset of $X$ and $V \supset A_{i} \cap U$ for some $U \in$ $p(\mathcal{U})\}, i=1$ or $i=2$, is an open filter base on $X$. Let $K=\left\{q\left(A_{1}\right), q\left(A_{2}\right)\right\} \cup$ $M(X) \backslash\{p(\mathcal{U})\}$, topologized so that it is the simple extension of $X$ with filter trace $\left\{q\left(A_{1}\right), q\left(A_{2}\right)\right\} \cup \mathbf{T} \backslash\{p(\mathcal{U})\}$. The resulting space $K$ is Hausdorff, and for every nonconvergent ultrafilter $\mathcal{C}$ on $X$, either $p(\mathcal{O}) \neq p(\mathcal{U})$ and $\mathcal{O}$ converges to $p(\mathcal{Y})$, or $\mathcal{O}$ converges to one of the points $q\left(A_{i}\right)$. Thus $K \in \mathcal{K}(X)$, so by (ii) there exists a continuous mapping $f: M(X) \rightarrow K$ with $f \mid X=i$. Since $M(X) \backslash\{p(\mathcal{U})\}=K \backslash\left\{q\left(A_{1}\right), q\left(A_{2}\right)\right\}$, it must also be true that for each $p\left(\mathcal{C}^{\mathcal{O}}\right) \neq p(\mathrm{U})$, one has $f\left(p\left(\mathcal{C}^{\complement}\right)\right)=p\left(\mathcal{C}^{\mathcal{O}}\right)$. On the other hand, since every Hausdorff continuous image of an absolutely closed space is absolutely closed, $f(M(X))$ must be a closed subset of $K$, so that $f(M(X))=K$. Thus $f(p(\mathcal{U}))=$ $q\left(A_{i}\right), i=1$ and $i=2$, which is impossible.

Therefore, there must exist a set $U \in p(\mathcal{U})$ such that, say, $A_{1} \cap U=\varnothing$. Then $A_{2}$ contains a member of $p(\mathcal{U})$, namely $\left(A_{1} \cup A_{2}\right) \cap U$, and hence $A_{2} \in p(\mathcal{U})$.

In the next theorem a relationship is established between $M(X)$ and $\beta X$ for certain completely regular Hausdorff spaces $X$. First a definition and two lemmas are needed.

A filter base $\mathcal{C}$ of open subsets of a space $X$ will be called completely regular provided that: (a) if $W$ is any open subset of $X$ containing a member of $\mathcal{C}$, then $W \in \mathcal{C}$; and (b) for each $U \in \mathcal{C}$ there exist $V \in \mathcal{C}$ and a continuous mapping $f: X \rightarrow[0,1]$ such that $f$ vanishes on $V$ and equals 1 on $X \backslash U$.

Some known properties of completely regular filters that we will use are listed in the next lemma. 
Lemma 3.3. On a topological space $X$ the following hold.

(i) If $\mathcal{F}$ and $\mathcal{G}$ are distinct maximal completely regular filters, then there exist $F \in \mathcal{F}$ and $G \in \mathcal{Y}$ with $F \cap G=\varnothing$.

(ii) If $\mathfrak{F}$ is a completely regular filter and $\mathcal{U}$ is an ultrafilter contain.

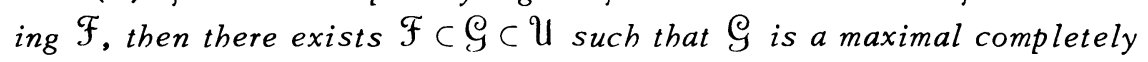
regular filter.

(iii) Let $\mathcal{F}$ be a maximal completely regular filter, and suppose that $U$, $V$ are open sets for which there is a continuous mapping $f: X \rightarrow[0,1]$ such that $f$ vanishes on $V$ and equals 1 on $X \backslash U$. Then either $U \in \mathcal{F}$ or there is a set $F \in \mathcal{F}$ with $V \cap F=\varnothing$.

The proof is straightforward.

Lemma 3.4。 Let $X$ be a Hausdorff space. The following are equivalent.

(i) If $\mathbf{T}$ is the family of all filter bases $\mathcal{F}$ on $X$ such that $\cap \mathcal{F}=\varnothing$ and $\mathcal{F}$ is a maximal completely regular filter on $X$, then there exists an e-compactification $K$ of $X$ whose filter trace on $X$ is $\mathbf{T}$.

(ii) There exists an e-compactification of $X$ each of whose trace filters is completely regular.

Proof. (i) implies (ii) is obvious. To establish (ii) implies (i), let

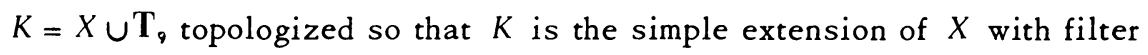
trace T. Then it follows easily from (i) and (ii) of Lemma 3.3 and (ii) above that $K$ is an e-compactification of $X$.

Notation. We will denote by $s M(X)$ the space which has the same points as those of $M(X)$ and whose topology has as a base the family of all regular open subsets of the space $M(X)$. A direct argument (which is a special case of a result in $[\mathrm{Ba}]$ ) shows that $s M(X)$ is a strict extension of $X$, i.e., if $V^{*}=V \cup\{p \mid V \in p\}$, then $\left\{V^{*} \mid V\right.$ is an open subset of $\left.X\right\}$ is a base for the topology on $s M(X)$.

A space $X$ will be called completely Hausdorff provided that for every pair of distinct points $x, y$ of $X$ there is a continuous mapping $f: X \rightarrow \mathbf{R}$ with $f(x) \neq f(y)$.

Theorem 3.5. Let $X$ be an e-compactifiable space. The following are equivalent.

(i) $\beta X$ exists and $\beta X=s M(X)$.

(ii) $s M(X)$ is compact.

(iii) $M(X)$ is a Urysohn space.

(iv) $X$ is completely Hausdorff, and each member of the filter trace of $M(X)$ is completely regular.

(v) $X$ is completely Hausdorff and has the following property: for every ultrafilter $\mathcal{U}$ on $X$, e-compactification $K$ of $X$, and element $\mathcal{F}$ of the filter 
trace of $K$, if $\mathcal{F} \subset \mathcal{U}$ then there is a completely regular filter $\mathcal{C}$ on $X$ with $\mathcal{F} \subset \mathcal{C} \subset \mathcal{U}$.

\section{Proof. Obviously (i) implies (ii).}

(ii) implies (i). If (ii) holds, then $s M(X)$ is a compactification of $X$, and so there exists a continuous mapping $g: \beta X \rightarrow s M(X)$ such that $g \mid X=i$. Since by (ii) of Theorem 3.2 there is also a continuous mapping $f$ of $M(X)$ and hence of $s M(X)$ into $\beta X$ with $f \mid X=i$, it follows that $g$ must be a homeomorphism.

(ii) and (iii) are equivalent, because by a result of Katětov [K], a space $Y$ is absolutely closed and Urysohn if and only if its associated space $s Y$ (defined in the same way as above) is compact Hausdorff.

(ii) implies (iv). Let $q \in M(X) \backslash X$, and let $\mathcal{C}(s \mathcal{C})$ denote the trace filter of $q$ in $M(X)(s M(X))$. Since $s M(X)$ is compact, $X$ and $s C$ are completely regular, and because $\left\{V^{*} \mid V\right.$ is an open subset of $\left.X\right\}$ is a base for $s M(X)$, $\mathcal{C}={ }_{s} \mathcal{C}$.

(iv) implies (iii). First let us observe that applying Lemma 3.4 and Theorem 3.2 (ii), one can easily prove that each trace filter of $M(X)$ is a maximal completely regular filter on $X$.

Now consider any two points $x_{1}, x_{2}$ of $M(X)$. By (iv) there exist open subsets $U_{i}$ and $V_{i}$ of $X$ and a continuous mapping $f_{i}: X \rightarrow[0,1], i=$ 1, 2, such that: (a) for each $i, x_{i} \in V_{i}^{*}$, and $f_{i}$ vanishes on $V_{i}$ and equals 1 on $X \backslash U_{i}$; and (b) $U_{1}^{*}$ and $U_{2}^{*}$ are disjoint. It follows from (a) and Lemma 3.3 (iii) that each $\mathrm{Cl}_{M(X)} V_{i}^{*} \subset U_{i}^{*}$. Thus $\mathrm{Cl} V_{1}^{*}$ and $\mathrm{Cl} V_{2}^{*}$ are disjoint closed neighborhoods of $x_{1}$ and $x_{2}$, and $M(X)$ is a Urysohn space.

The proof that (iv) and (v) are equivalent is straightforward.

The next result is an immediate consequence of Theorems 3.5 and 1.1 and the proof of Urysohn's lemma.

Corollary 3.6. Let $X$ be an e-compactifiable space having the following property: for every pair of regular open sets $U, V$ such that $\bar{V} \subset U$, there exists an open set $W$ such that $\bar{V} \subset W \subset \bar{W} \subset U$. Then $\beta X=s M(X)$.

Several well-known examples can be used to show that the converse of Corollary 3.6 is false.

Example 3.7. Let $T$ denote any one of the regular but not completely regular spaces in $[\mathrm{T}]$. [Hew, the Arens space], [BS], $[\mathrm{S} 2, \S 3]$, or $[\mathrm{S} 1$, the space $X$ in 1.8], and let $X$ be the space obtained by removing from $T$ the point(s) at which $T$ fails to be completely regular. Then $X$ is completely regular and Hausdorff and does not satisfy the hypothesis of Corollary 3.6, but lengthly arguments, not too different from ones in [BS] and [J2], show that (v) of Theorem 3.5 does hold.

It should be mentioned that in $[\mathrm{H}]$ Hechler proved that several of the 
spaces $T$ in Example 3.7 are not e-compactifiable. One can also prove that none of the other spaces $T$ given is e-compactifiable.

We conclude by raising the following

Question. Does there exist an e-compactifiable space $X$ for which $s M(X)$ fails to be compact?

If the answer is no then every e-compactifiable space is completely regular, and if the answer is yes then by Theorem 3.5 there are an e-compactifiable space $X$ and a point $p \in M(X)$ for which the $e$-compactifiable space $X \cup\{p\}$ is not completely regular.

If $T$ is the space $X(\pi)$ in $[S 1,1.8]$ or one of the regular but not completely regular spaces in [J1] or $[S 2, \$ 4]$, and if $X$ is the space obtained by removing the point(s) at which $T$ is not completely regular, then the author does not know if $s M(X)$ is compact.

\section{REFERENCES}

[Ba]. B. Banaschewski, Uber Hausdorffsch-minimale Erweiterung von Rämen, Arch. Math. 12 (1961), 355-365. MR 25 \#5490.

[BPS]. M. P. Berri, J. R. Porter and R. M. Stephenson, Jr., A survey of minimal topological spaces, General Topology and its Relations to Modern Analysis and Algebra, III (Proc. Conf., Kanpur, 1968), Academia, Prague, 1971, pp. 93-114. MR 43 \# 3985.

[BS]. M. P. Berri and R. H. Sorgenfrey, Minimal regular spaces, Proc. Amer. Math. Soc. 14 (1963), 454-458. MR 27 \#2949.

[Bo]. N. Bourbaki, Espaces minimaux et espaces complètement séparés, C. R. Acad. Sci. Paris 212 (1941), 215-218. MR 3, 136.

[H]. S. H. Hechler, On a notion of weak compactness in non-regular spaces, Studies in Topology, N. M. Stavrakas and K. R. Allen, Editors, Academic Press, New York, 1975, pp. 215-237.

[Hew]. E. Hewitt, On two problems of Urysohn, Ann. of Math. (2) 47 (1946), 503-509. MR 8, 165.

[J1]. F. B. Jones, Moore spaces and uniform spaces, Proc. Amer. Math. Soc. 9 (1958), 483-486. MR 20\#277.

[J2]. - Hereditarily separable, non-completely regular spaces, Proc.

Topology Conference Virginia Polytechnic Inst. and State Univ., March 22-24, 1973, Lecture Notes in Matho, no. 375, Springer-Verlag, Berlin and New York, 1974, pp. 149-152.

[K]. M. Katětov, Über H-abgeschlossen und bikompakt Räume, Časopis, Pěst. Mat. Fys. 69 (1940), 36-49. MR 1, 317.

[S1]. R. M. Stephenson, Jr., Minimal first countable Hausdorff spaces, Pacific J. Math. 36 (1971), 819-825. MR 44 \#5916.

[S2]. R. M. Stephenson, Jr., Two R-closed spaces, Canad. J. Math. 24 (1972), 286-292. MR 45 \#7665.

[T]. A. Tychonoff, Über die topologische Erweiterung von Räumen, Math. Ann. 102 (1930), 544-561. 